\section{Hepatitis B}

\section{Immunmodulator mit Potenzial}

Auf dem „Liver Meeting 2017“ der AASLD in Washington DC präsentierte AiCuris die Ergebnisse einer präklinischen Studie zum Potenzial des proprietären Immunmodulator AIC649 zur Heilung von Infektionen, die durch das Hepatitis B-Virus (HBV) verursacht werden (Poster LB-22). Darüber hinaus sollte die Wirksamkeit und Sicherheit von AIC649 als Monotherapie und in Kombination mit dem direkt wirkenden Virustatikum Entecavir (ETV) untersucht werden.

In der Studie wurden Waldmurmeltiere, die chronisch mit dem Waldmurmeltier-Hepatitis-Virus (WHV) infiziert waren, 36 Wochen entweder mit AIC649 als Monotherapie oder als Kombinationstherapie mit Entecavir (ETV) behandelt. Die Wirksamkeit von AIC649 und ETV jeweils als Mono- sowie von AIC649 und ETV als Kombinationstherapie wurde im Vergleich zu Placebo ermittelt. Neben der antiviralen Wirkung wurden die durch die Behandlung hervorgerufenen Veränderungen in der Antigenämie, in den immunologischen Parametern sowie in der Induktion der WHsAg-AntikörperSerokonversion (erstmaliges Auftreten der Antikörper im Serum) evaluiert.

AIC649 induzierte im Waldmurmeltiermodell für chronische Hepatitis B eine physiologische Immunreaktion. Die Studienergebnisse zeigten einen langanhaltenden Verlust der spezifischen Hepatitis-B-Oberflächenantigene WHsAg (Woodchuck Hepatitis B surface antigen) sowie die Bildung von anti-WHsAg-Antikörpern. In Kombination mit dem Therapiestandard Entecavir (ETV) verstärkte AIC649 die therapeutische Wirkung der Behandlung signifikant.

Die Ergebnisse der präklinischen Studie stützen das Potenzial von AIC649, einem vielversprechenden Kandidaten aus unserer breiten Entwicklungspipeline von antiviralen Substanzen, bei HBV-infizierten Patienten eine funktionelle Heilung $\mathrm{zu}$ induzieren“, sagte Holger Zimmermann, CEO von AiCuris Anti-infective Cures. red

Nach Informationen von AiCuris

Auf dem Weg zur Präzisionsmedizin

\title{
Gastrointestinale Tumoren: Neues zur Checkpoint-Hemmung
}

Die Immunonkologie stellt einen wichtigen Schritt auf dem Weg zur Präzisionsmedizin dar. Welche Chancen bietet sie bei gastrointestinalen Tumoren?

Die Immunonkologie sei auch für die Behandlung gastrointestinaler Tumoren vielversprechend, erklärte Prof. Florian Lordick, Leipzig. So hatten Patienten mit einem Karzinom des Magens und des gastroösophagealen Übergangs in einer randomisierten, doppelblinden, placebokontrollierten Phase-III-Studie nach zwölfmonatiger Behandlung mit Nivolumab $\left(\mathrm{Opdivo}^{\circledR}\right)(\mathrm{n}=330)$ im Vergleich $\mathrm{zu}$ Placebo $(\mathrm{n}=163)$ einen deutlichen Überlebensvorteil (26,2 vs. 10,9\%) sowie ein signifikant verbessertes progressionsfreies Überleben (1,61 vs. 1,45 Monate). Das Risiko für einen Krankheitsprogress war unter Nivolumab niedriger als unter
Placebo (Hazard Ratio 0,60, p < 0,0001) [Kang YK et al. Lancet. 2017; https://doi. org/10.1016/S0140-6736(17)31827-5].

Bei Patienten mit Kolorektalkarzinom, deren Tumoren Defekte in der DNA-Mismatch-Reparatur oder eine hohe Mikrosatelliteninstabilität aufwiesen, wurde die Kombination aus Nivolumab plus Ipilimumab (Yervoy ${ }^{\circledast}$ ) untersucht. Die Interimsdaten dieser Phase-II-Studie ergaben eine Ansprechrate von 54,8\%; die 9-Monats-Rate für das Gesamtüberleben betrug 87,6\% [Andre T et al. J Clin Oncol 2017; 35(15, Suppl): 3531].

Kathrin Strobel

Symposium „Immunonkologie 2017: Auf dem Weg zur Präzisionsmedizin", DGHO-Jahrestagung, 1.10.2017, Stuttgart; Veranstalter: Bristol-Myers Squibb Pressekonferenz

\section{Leberzellkarzinom}

\section{Neue Sequenztherapie bringt Fortschritte}

Bisher gab es trotz zahlreicher Studien keine Behandlungsmöglichkeit in der Zweitlinie beim HCC für Patienten, die unter der Erstlinientherapie mit Sorafenib (Nexavar ${ }^{\varpi}$ ) progredient wurden, erläutert PD Dr. Marcus-Alexander Wörns, Mainz, auf einer Pressekonferenz am Rande der DGHO-Jahrestagung. Diese Therapielücke wird nun von dem oralen Multi-Kinase-Inhibitor Regorafenib (Stivarga ${ }^{\circledR}$ geschlossen. Basis für die EU-Zulassung waren die Ergebnisse der internationalen, multizentrischen, placebokontrollierten Phase-III-Studie RESOR-CE [Bruix J et al. Lancet 2017; 389: 56 - 66]. In RESORCE wurde Regorafenib an 573 randomisierten Patienten mit inoperablem HCC untersucht, die unter Sorafenib progredient geworden waren. Alle Patienten erhielten neben Regorafenib oder Placebo die bestmögliche unterstützende Behandlung (BSC). In der Verumgruppe verbesserte sich das mediane OS signifikant im Vergleich zum Kontrollarm von 7,8 auf 10,6 Monate, was eine relative Reduktion des Mortalitätsrisikos von $37 \%$ über den Studienzeitraum bedeutet $(\mathrm{HR}=0,63$; $95 \%-K I$ : 0,50-0,79; p<0,0001). Die Nebenwirkungen entsprachen dem bekannten Sicherheitsprofil von Regorafenib. Als häufigste behandlungsbedürftige Nebenwirkungen traten Hand-Fuß-Hautreaktionen, Diarrhö, Fatigue sowie Bluthochdruck auf. -Besonders positiv bewertete Wörns die Ergebnisse einer gepoolten Analyse, die zeigte, dass Patienten mit der Sequenztherapie Sorafenib-Regorafenib ein medianes OS von 26 Monaten erreichen konnten und damit deutlich länger lebten als Patienten unter der Sequenz Sorafenib-Placebo mit einem medianen OS von 19,2 Monaten [Kudo M. Liver Cancer 2017; 6: 177 -84) Regorafenib wird nicht mehr in Deutschland vertrieben, ist aber weiterhin in der EU zugelassen und kann somit in den zugelassenen Indikationen bei entsprechender Indikationsstellung verordnet werden.

red

Nach Information von Bayer Vital 